FERMILAB-TM-1753

\title{
Technical Memo on New Results on CsI Photocathodes: Enhancement and Aging
}

\author{
D. F. Anderson, S. Kwan \\ Fermi National Accelerator Laboratory \\ P.O. Box 500, Batavia, Illinois 60510 \\ B. Hoeneisen \\ Universidad San Francisco de Quito \\ P.O. Box 17-1200841, Quito, Ecuador \\ V. Peskov \\ CERN/World Lab \\ Geneva, Switzerland
}

September 1991 
TM-1753

September 1991

\title{
Technical Memo on \\ New Results on \\ CsI Photocathodes: \\ Enhancement and Aging
}

\author{
D.F. Anderson, B. Hoeneisen 1 , S. Kwan, and V. Peskov ${ }^{2}$ \\ Particle Detector Group \\ Fermi National Accelerator Laboratory \\ Batavia IL 60510 U.S.A.
}

\begin{abstract}
It appears that there are 4 processes involved in the enhancement and aging of a CsI or CsI-TMAE photocathode: water absorption, charging up of the photocathode, a self annealing aging, and a permanent aging. The evidence for these processes are presented.
\end{abstract}

${ }^{1}$ Universidad San Francisco de Quito, P.O. Box 17-1200841, Quito, Ecuador

${ }^{2}$ CERN/World Lab, Geneva Switzerland 


\section{Introduction}

The use of pure CsI or CsI-TMAE as a photosensitive element in wire and parallel plate chambers has been recently demonstrated [1-3]. Detectors with this solid photocathode have a better time resolution than the conventional detectors with photosensitive vapors. In fact it is a new type of detector, which some authors call gaseous photomultipliers [4]. However large scale applications of this device have been restricted due to the aging of the CsI photocathode [3-6]. Some authors $[5,7,8]$ have observed enhancement of the quantum efficiency of the CsI photocathode under some conditions which in principle could compete with aging.

In this Technical Memo we report our latest results on the aging and enhancement properties of the CsI and CsI-TMAE photocathodes. It is intended to be an informal (but referential) communication to those working in the field.

\section{Experimental set-up}

The parallel plate avalanche chamber used for this study is shown in fig. 1 . It is similar to the one described in references 3 and 5, except that it contains a cathode that can be cooled or heated between -20 and $+100^{\circ} \mathrm{C}$. The chamber was evacuated to a pressure of $10^{-4}$ when operated in the diode mode. Measurements with gain were also made at pressures of 10 Torr to $1 \mathrm{~atm}$. The quantum efficiency of the photocathode is measured relative to the known efficiency of TMAE gas as described in reference 3 . The ultraviolet light source used is a $\mathrm{Hg}$ lamp with its intensity monitored by a photodiode. For most of the measurements the UV light passed through a filter at $\lambda \approx 185 \mathrm{~nm}$ or through a monochrometer. The area of the illuminated spot on the photocathode for all measurements was $113 \mathrm{~mm}^{2}$. Several photocathodes were tested: 0.5 and $0.015 \mu \mathrm{m}$ pure CsI, and 2 and $0.1 \mu \mathrm{m}$ of CsI with an adsorbed TMAE layer as described in reference 3.

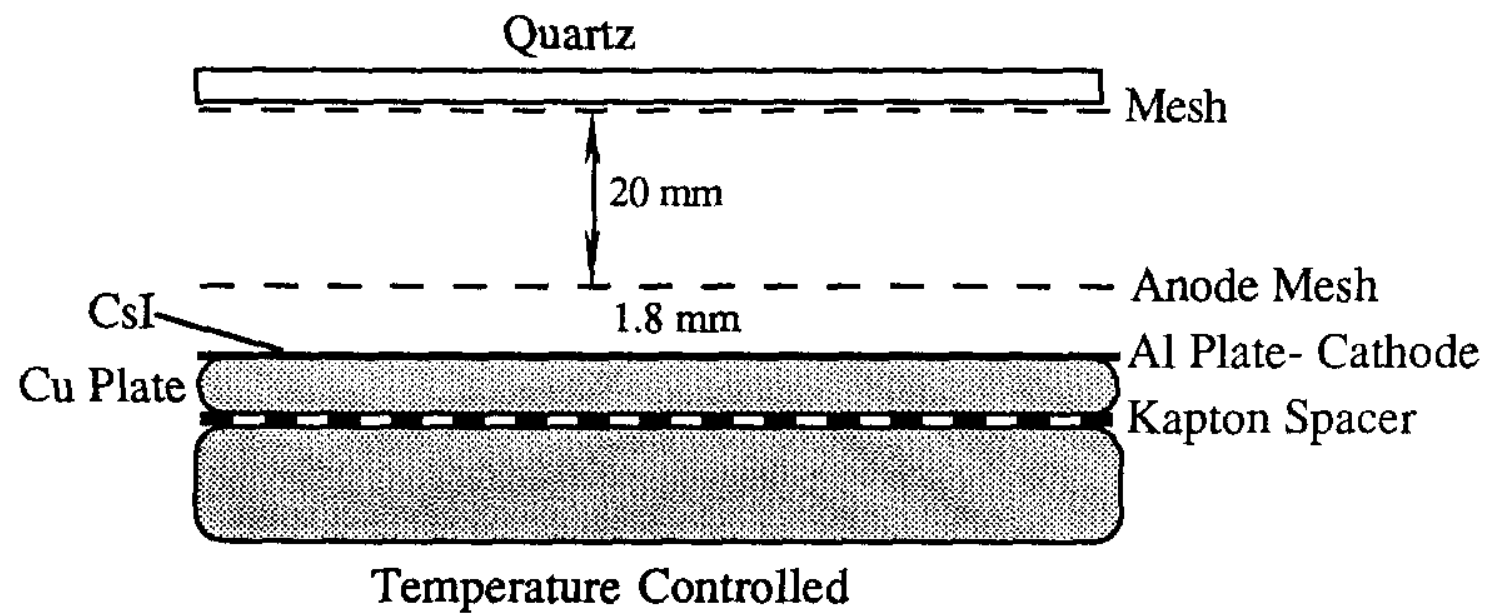

Figure 1. Parallel plate avalanche chamber with solid photocathode. 


\section{Measurements}

We observed the current (which is proportional to the quantum efficiency, QE, of the photocathode) in the chamber for pressures ranging from $10^{-4}$ to $1 \mathrm{~atm}$ and for gas gains that varied from 1 to $10^{6}$. A typical measurement for a $0.1 \mu \mathrm{m}$ thick CsI-TMAE photocathode with 20.4 Torr of methane and a gas gain of 262 is shown in Fig. 2. From time 0 to $t_{1}$ the photocathode is illuminated with intense UV light. The current (and QE) decreased as shown. At time $t_{1}$ the light was blocked and the quantum efficiency restores in time as shown by momentarily unblocking the UV light at times $t_{2}-t_{5}$. These results are typical at room temperature operation at all pressures and gains studied.

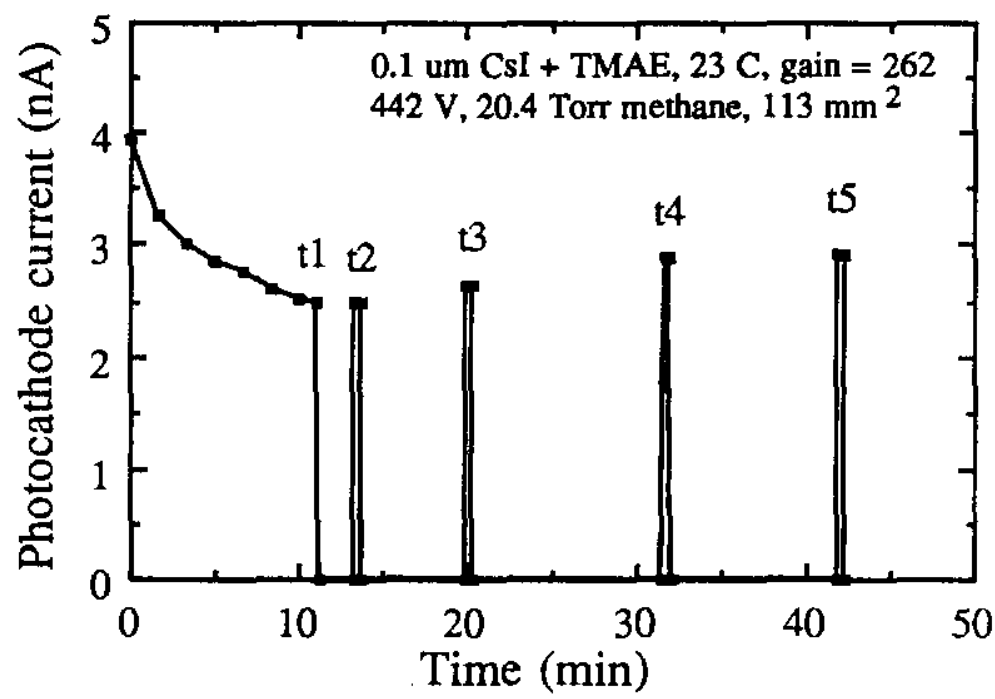

Figure 2. Current of a CsI-TMAE photocathode as a function of time for a typical measurement. The gas gain is 262 and the pressure is 20.4 Torr of methane. The UV light is interrupted at time $t 1$, and reestablished for a moment at times $t_{2}-t_{5}$.

Fig. 3 shows the response of a pure CsI photocathode $0.015 \mu \mathrm{m}$ thick in vacuum with a collection voltage of $11 \mathrm{~V}$. We observe the same degradation of the $\mathrm{QE}$ as seen in the early part of fig. 2 at room temperature. At time $t_{1}$ the photocathode heater is turned on. The efficiency initially decreases by a few percent, then raises significantly, and finally slowly decreases again when the temperature stabilizes. The rate of aging at $80^{\circ} \mathrm{C}$ is much less than for room temperature. A similar enhancement by a factor $\approx 1.7$ is observed when heating a freshly evaporated photocathode to $97^{\circ} \mathrm{C}$. This enhancement is not lost by a return to room temperature or by pumping to $10^{-3}$ Torr.

It is interesting to note that when a similar measurement to the one in fig. 3 was made with pure $\mathrm{He}$ as the counter gas at a gain $>1$, at the point that the heater was turned on there was a sudden, transient increase in the current. This is most likely due to the outgassing of something from the photocathode. The gas, having a lower ionization potential than the 
first excited state of $\mathrm{He}$, causes an increase in current by the Penning effect. This effect is seen for both CsI and CsI+TMAE photocathodes.

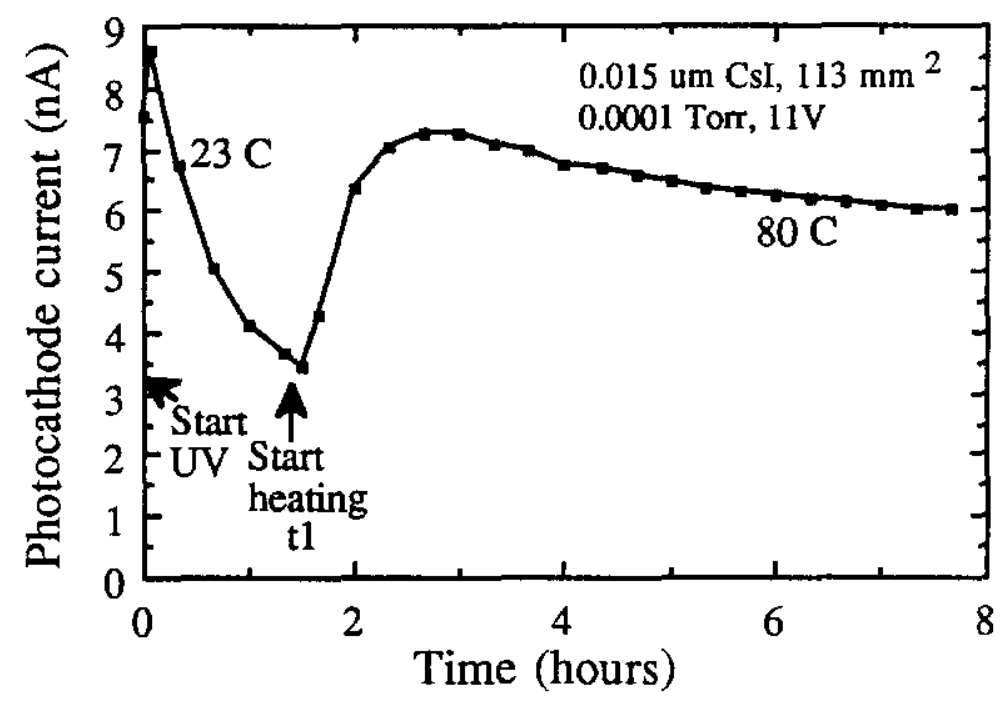

Figure 3. Current as a function of time for a CsI photocathode. At time $t_{1}$ the photocathode is heated to $80^{\circ} \mathrm{C}$.

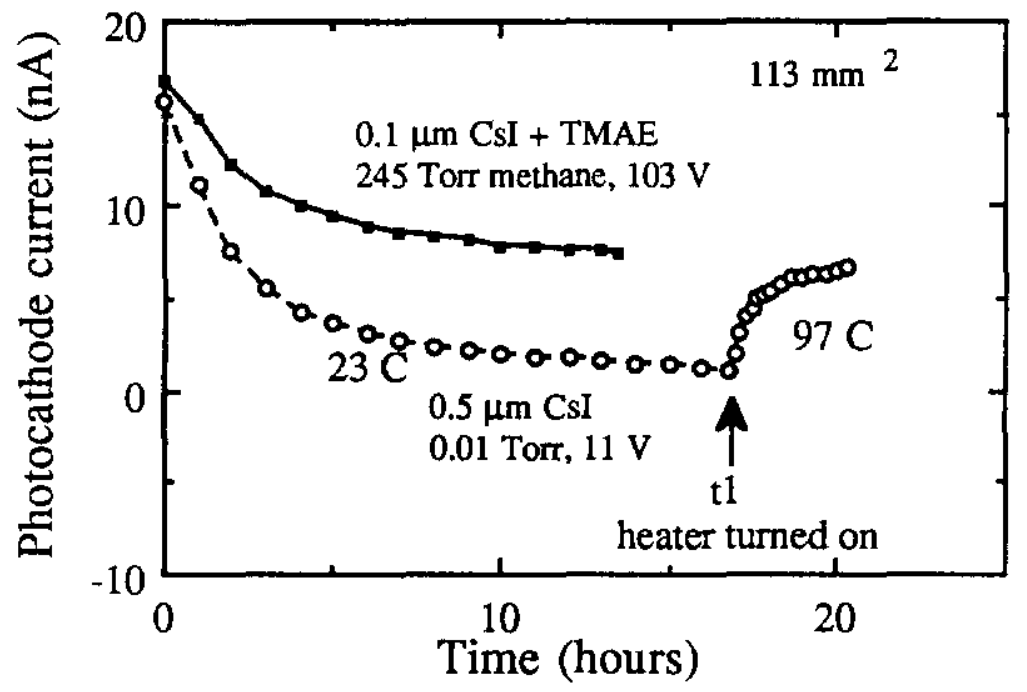

Figure 4. Currents as a function of time for a $0.1 \mu \mathrm{m}$ thick CsI+TMAE and a 0.5 $\mu \mathrm{m}$ thick CsI photocathode.

In fig. 4 we compare the aging properties of a particular CsI photocathode to those of a particular CsI-TMAE photocathode, both at room temperature. Initially the drop in current is similar for both photocathodes, but with the current of the CsI-TMAE photocathode showing a smaller degradation The enhancement of the $\mathrm{QE}$ of the CsI photocathode, when it is heated, is also shown. 


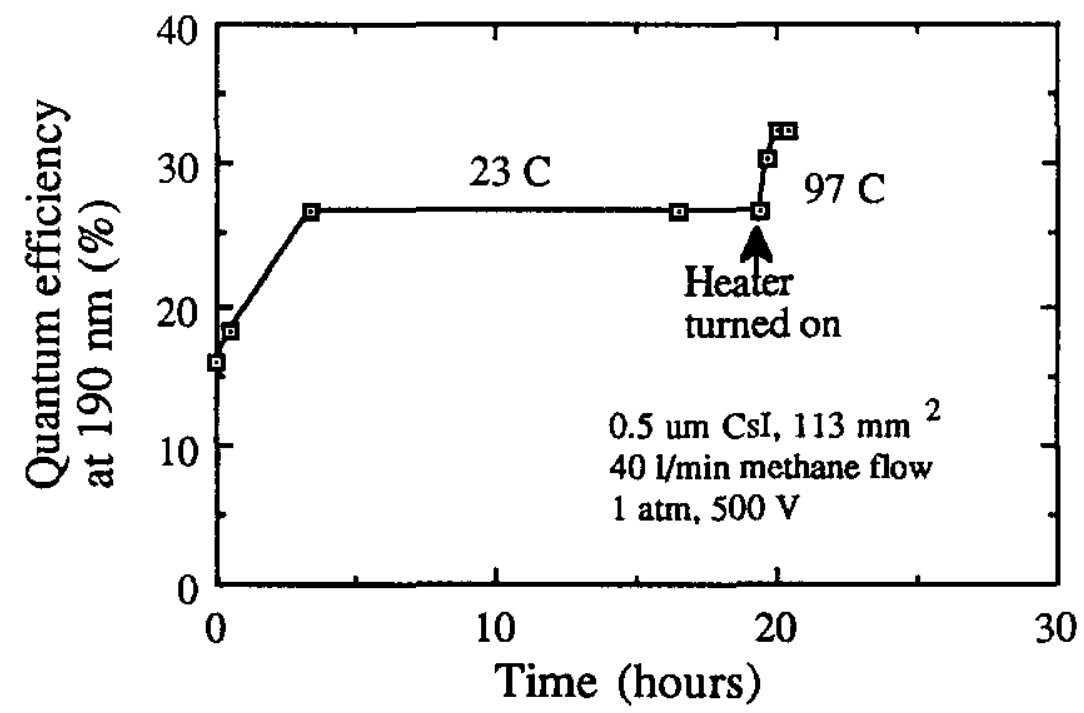

Figure 5. QE as a function of time of a freshly deposited , $0.5 \mu \mathrm{m}$ thick CsI photocathode with a $40 \mathrm{l} / \mathrm{min}$ flow of methane. At 20 hours the photocathode is , heated to $97^{\circ} \mathrm{C}$.

There is one other measurement that also relates to the phenomenon of enhancement. Fig.5 which shows the $\mathrm{QE}$ at $\lambda=190 \mathrm{~nm}$ of a freshly evaporated $0.5 \mu \mathrm{m}$ CsI photocathode operated at $1 \mathrm{~atm}$ with a $40 \mathrm{l} / \mathrm{min}$ flow for pure methane, initially at $23^{\circ} \mathrm{C}$. The $\mathrm{QE}$ increases with time a and plateau is reached at about $27 \%$. This is a similar result to that seen by others [8] but it is achieve on a shorter time scale. At $t=20$ hours the cathode was heated to $97^{\circ} \mathrm{C}$ and there is an almost instantaneous jump in $\mathrm{QE}$ to about $34 \%$. This result will be interpreted in the next section.

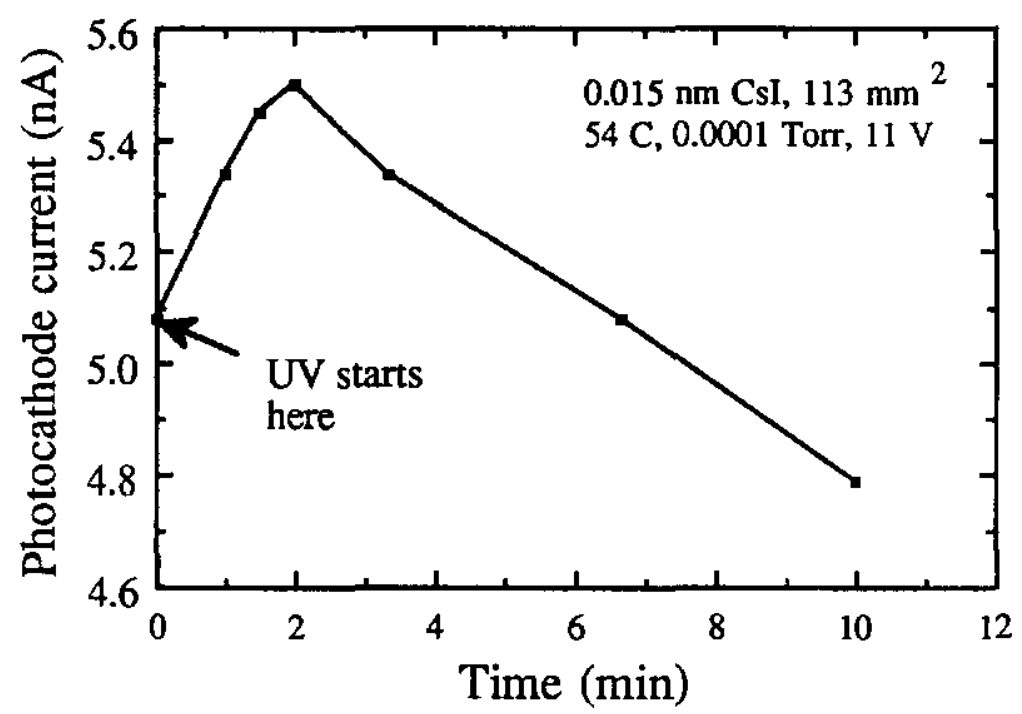

Figure 6. Current as a function of time for a CsI photocathode showing the initial enhancement seen in almost all measurements. 
In fig. 6 we present the initial enhancement of the quantum efficiency when the UV light is unblocked and when the chamber is operated at a relatively high vacuum ( $10^{-4}$ Torr) and a gain of 1 . There is an initial increase in current (QE) before the degradation. This phenomenon is same as the one reported in an earlier work [5]. See fig. 3 for another example of this effect.

\section{Working Hypotheses}

Many measurements have been made. Not all of the results can be presented in this Technical Memo. We have developed a working hypotheses which seems to explain our results to date and will be used to direct future research. Our hypotheses is that there are four major phenomena involved:

1. Water absorbed by the photocathode lowers the QE. This water can be removed by vacuum, heating, or a flow of dry gas and explains the enhancement seen in fig. 5. These results explain the enhancement that Séguinot et al.[8] see with the flowing of clean methane.

2. A charging up of the photocathode, increasing the QE. This is seen in figs. 3 and 6 . The enhancement seen by Anderson and Kwan [5] now appears to be due to charging up of the photocathode.

3. An unidentified reversible process of aging and recovery of the surface of the photocathode. It is induced by UV photons and positive ions of the avalanche. At a gain of 1 the charge saturates this process at $\approx 5 \mu \mathrm{C} / \mathrm{mm}^{2}$, equivalent to one elementary charge per $3 \AA^{2}$. The recovery rate increases with temperature, yielding a higher equilibrium $\mathrm{QE}$ with higher temperature. As one would expect, the aging process is related to the current intensity, with a higher equilibrium QE for lower currents. See figs. 2 and 3.

4. An unidentified irreversible aging process that determines the long term rate of decrease of efficiency after the reversible phenomena have reach equilibrium.

\section{Acknowledgement:}

The authors would like to thank SSClab, Professor Zichichi, and the World Lab for support of this work. We would also like to thank the technical support of Sharon Austin, Eileen Hahn and Jerry Zimmerman. 


\section{References}

[1] G. Charpak et al, "New Scintillators and Photosensitive Gaseous Detectors", Proceedings of the Symposium on Particle Identification at High Luminosity Hadron Colliders, Fermilab, Batavia, 5-7 April 1989.

[2] V. Dangendorf et al, NIM A289 (1990) 322.

[3] B. Hoeneisen, D.F. Anderson and S. Kwan, NIM A302 (1991) 447-454.

[4] V. Dangendorf et al, "Progress in Ultra-fast CsI Photocathode Gaseous Imaging Photomultipliers", preprint WIS 91/90 Apr-PH

[5] S. Kwan and D.F. Anderson,"A Study of the CsI-TMAE Photocathode", submitted to NIM.

[6] G. Charpak et al, "New Photocathodes for Fast Gaseous Detectors", Preprint . CERN PPE/90-185.

[7] D.C. Imrie et al, "Development of Large Area Air Stable Photocathode for the Near Ultraviolet", preprint BRU/DDG/108 Sept 1990.

[8] J. Seguinot et al, NIM A297 (1990) 133.

[9] G. Charpak et al, "Investigation of Operation of a Parallel Plate Avalanche Counter with a CsI Photocathode", preprint CERN-PPE/91-47. 